\title{
EFECTO DE LA COBERTURA EN LAS PROPIEDADES DEL SUELO Y EN LA PRODUCCIÓN DE FRÍJOL IRRIGADO
}

\section{MULCHING AND ITS EFFECTS ON SOIL PROPERTIES AND PRODUCTION OF IRRIGATED BEANS}

\author{
Carolina María Sánchez-Sáenz¹, Zigomar Menezes de Souza², Edson Eiji Matsura³ \\ Natália Regina Salomão de Freitas ${ }^{4}$
}

\begin{abstract}
${ }^{1}$ Ingeniera Agrícola, M.Sc. Profesor Auxiliar. Facultad de Ingeniería, Programa de Ingeniería Agrícola, Universidad Nacional de Colombia. Cra. 30 No. 45 - 03, Bogotá, Colombia. E-mail: cmsanchezs@unal.edu.co. Autor para correspondencia. ${ }^{2}$ Ingeniero Agrónomo, Profesor Doctor, Depto. de Suelo y Agua, Facultad de Ingeniería Agrícola, Universidad Estatal de Campinas (UNICAMP), Ciudad Universitaria Zeferino Vaz, Caja Postal: 6011, Campinas, SP, CEP 13083-875 - E-mail: zigomarms@feagri.unicamp.br ${ }^{3}$ Ingeniero Agrónomo, Profesor Titular, Depto. de Máquinas Agrícolas, Facultad de Ingeniería Agrícola (UNICAMP) Ciudad Universitaria Zeferino Vaz, Caja Postal: 6011, Campinas, SP, CEP 13083-875.E-mail: matsura@feagri.unicamp.br ${ }^{4}$ Estudiante en Ingeniería Agrícola Universidad Estatal de Campinas/UNICAMP - Ciudad Universitaria Zeferino Vaz, Caja Postal: 6011, Campinas, SP, CEP 13083-875. E-mail:natalia.freitas@feagri.unicamp.br
\end{abstract}

Rev. U.D.CA Act. \& Div. Cient. 13 (2): 41-50, 2010

\section{RESUMEN}

Dentro de las principales características buscadas, las plantas utilizadas como cobertura deben proteger el suelo y mejorar sus propiedades físicas, químicas y biológicas para el cultivo subsecuente. Por tanto, el objetivo de este trabajo fue evaluar el efecto de las cantidades de cobertura y dosis de nitrógeno mineral en las propiedades físicas y en la temperatura del suelo, en el contenido de humedad del suelo y en la producción de frijol durante el ciclo del cultivo, en siembra directa. El ensayo fue implementado mediante un diseño experimental de bloques aleatorios, con tres repeticiones y esquema factorial $3 \times 4$. Las dosis de nitrógeno fueron ausencia de $\mathrm{N}$, $50 \mathrm{~kg} \cdot \mathrm{ha}^{-1}$ y $120 \mathrm{~kg} \cdot \mathrm{ha}^{-1}$ y las cantidades de cobertura fueron ausencia, 4,0t-ha ${ }^{-1}, 6,0 \mathrm{t} \cdot \mathrm{ha}^{-1}$ y $10,0 \mathrm{t} \cdot \mathrm{ha}^{-1}$. En los tratamientos con aplicación de residuos $\left(4,0 ; 6,0\right.$ y $\left.10,0 \mathrm{t} \cdot \mathrm{ha}^{-1}\right)$ hubo mayor sustento en el contenido de agua en el suelo del ciclo del cultivo de fríjol irrigado, comparado con la condición de superficie del suelo descubierto. El tratamiento de $10,0 \mathrm{t} \cdot \mathrm{ha}^{-1} \mathrm{de}$ residuos de cobertura promovió disminución de la resistencia del suelo a la penetración, aumento en la humedad del suelo y ocasionó menores diferencias de temperatura en el ciclo del cultivo de fríjol irrigado, comparado con el tratamiento testigo. El tratamiento de $10,0 \mathrm{t} \cdot \mathrm{ha}^{-1}$ de residuo y $120 \mathrm{~kg} \cdot \mathrm{ha}^{-1}$ de nitrógeno proporcionó la mayor producción de fríjol.

Palabras clave: Siembra directa, humedad, temperatura, propiedades suelo.

\section{SUMMARY}

Plant residues employed as mulching material are one of the main factors that contribute to the improvement of the physical, chemical and biological soil proprieties for subsequent crops. Therefore, the objective of this study was to evaluate the effect of the combination of different amounts of residues and mineral nitrogen on soil physical attributes, soil temperature, soil water content and grain yield during the whole cycle of a bean crop under the no-tillage system. The experiments were organized in randomized blocks with three replications using a $3 \times 4$ factorial design. The nitrogen levels were zero, $50 \mathrm{~kg} \cdot \mathrm{ha}^{-1}$ and $120 \mathrm{~kg} \cdot \mathrm{ha}^{-1}$ and the straw mulch amounts were zero, $4.0 \mathrm{t} \cdot \mathrm{ha}^{-1}, 6.0 \mathrm{t} \cdot \mathrm{ha}^{-1}$ and $10.0 \mathrm{t} \cdot \mathrm{ha}^{-1}$. The treatments that received residues applications presented greater maintenance of soil water content throughout the whole cultivation cycle when compared to the soil without mulching. The treatment of $10.0 \mathrm{t} \cdot \mathrm{ha}^{-1}$ amount of mulching on the surface promoted reduction in the soil penetration resistance, increase in the soil water content and minor differences of temperature throughout the cycle of the culture. The treatment of $10,0 t^{-} \mathrm{ha}^{-1}$ of residues and nitrogen promoted an increase in the yield of beans.

Key words: No-tillage, water contents, temperature, soil properties. 


\section{INTRODUCCIÓN}

La cobertura del suelo pasa a ser uno de los factores más eficientes en la minimización de los efectos indeseables, que se derivan de la exploración de los suelos agrícolas, debido, especialmente, a la acción protectora proporcionada por los residuos orgánicos dejados por los cultivos, los cuales, actúan interceptando las gotas de lluvia y disipando su energía cinética (Saturnino $\mathcal{E}$ Landers, 1997). La cobertura es un factor para el éxito de la producción agrícola en la siembra directa, principalmente, en lo referente a la economía de agua. Dentro de las principales características buscadas, las plantas utilizadas deben proteger el suelo y mejorar sus propiedades físicas, químicas y biológicas para el cultivo siguiente (Campos et al. 1995; Furlani et al. 2008).

El sistema de labranza del suelo, además de influir en la distribución del residuo depositado en la superficie del suelo, modifica la relación masa-volumen, la cantidad de agua en el suelo, el contenido de materia orgánica, la estructura y la temperatura del suelo, entre otros (Costa et al. 2003; Oliveira et al. 2005). Cultivos que fueron desarrollados sobre siembra directa, después de haber sido cultivados con labranza convencional o reducida, presentaron en los primeros años, después del cambio del sistema de cultivo, un estado de compactación más elevado, comparado con cultivos que continuaron siendo labrados tradicionalmente (Genro Júnior et al. 2004).

La temperatura del suelo ejerce marcada influencia sobre los procesos físicos, químicos y biológicos que en él ocurren y varía, considerablemente, con el tipo de planta. La humedad del suelo desempeña, e igual manera, un importante papel en la determinación de la cantidad de calor utilizado en el proceso de evaporación del agua existente en él (Derpsch et al. 1991; Furlani et al. 2008). Las temperaturas del suelo son influenciadas por sus coberturas naturales y, especialmente, por residuos orgánicos u otros tipos de coberturas protectoras, aplicadas en su superficie. En periodos de calor mantienen la superficie del suelo más fresca que en los casos en que no existe cobertura. En contraste, en los periodos fríos de inverno, funcionan como moderadores de las rápidas caídas de temperatura (Salton \& Mielniczuk, 1995).

Estudiando los efectos de la cobertura vegetal en la temperatura y la humedad del suelo en el cultivo de maíz, Lal (1974) constató que la adición de residuos de arroz disminuyó la temperatura máxima en las tres profundidades estudiadas, en comparación con la parcela con suelo descubierto, constatando que el residuo aumentó la capacidad de retención de agua del suelo y que las diferencias de humedad también decrecen en profundidad. Bragagnolo $\&$ Mielniczuk (1990) evaluando el efecto de la cobertura del suelo con residuos de cosechas en la temperatura y humedad del suelo, por medio de la aplicación de diferentes dosis de residuo de trigo, verificaron que $7,5 \mathrm{t} \cdot \mathrm{ha}^{-1}$ de residuo aplicado contribuyó para la reducción de la temperatura del suelo en $8,5^{\circ} \mathrm{C}$, además de retener $10 \%$ más de agua que en el suelo descubierto, no afectando la germinación de la soya.

En la región de Campinas, uno de los factores limitantes para la siembra directa es la permanencia de la cobertura en el suelo, dado que los factores climáticos, como la alta temperatura, favorece el rápido proceso de descomposición, siendo necesario estudiar este factor, para adoptar la especie adecuada a las condiciones presentes en la zona. Por tanto, el objetivo de este trabajo fue determinar el efecto de las cantidades de cobertura del suelo y dosis de nitrógeno mineral en las propiedades físico-hídricas del suelo, temperatura y humedad del mismo y en la producción de fríjol irrigado, durante el ciclo del cultivo en siembra directa.

\section{MATERIALES Y MÉTODOS}

La investigación fue realizada en el área Experimental de la Facultad de Ingeniería Agrícola de la UNICAMP, situada en el municipio de Campinas (SP), con coordenadas geográficas de $22^{\circ} 48^{\prime} 57^{\prime \prime}$ de latitud sur y $47^{\circ} 03^{\prime} 33^{\prime \prime}$ de longitud oeste. El suelo, de acuerdo con la nomenclatura actual de (EMBRAPA (1999), pertenece a la clase Rhodic Haplorthox, USA; Rhodic Ferralsol, FAO. El tipo climático, según Köppen 1936), es una transición entre los tipos Cwa y Cfa, indicando clima tropical de altitud con invierno seco y verano húmedo.

El experimento fue realizado en un diseño de bloques aleatorios, con tres repeticiones y esquema factorial $3 \times 4$. Los tratamientos constaron de tres niveles de nitrógeno $\left(0 \mathrm{~kg} \cdot \mathrm{ha}^{-1}\right.$,

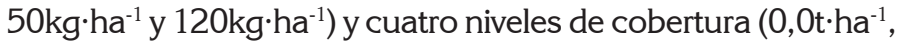
4,0t·ha ${ }^{-1}, 6,0 \mathrm{t} \cdot \mathrm{ha}^{-1}$ y 10,0 tha $\left.^{-1}\right)$. Cada parcela presentó $15 \mathrm{~m}^{2}$, para un total de $180 \mathrm{~m}^{2}$ de área útil por bloque.

Antes de 2004, el área era manejada con un sistema convencional (arado de discos y niveladoras); la fertilización, se realizaba con formulaciones establecidas y la cobertura con nitrógeno a los 25 días. Desde el año agrícola de 2004/2005, se maniobra con el sistema de siembra directa, el material de cobertura es desecado con herbicida y después de ese manejo es realizada la siembra con máquinas específicas, para la siembra directa. La secuencia de cultivos manejado es maíz, en verano y nabo forrajero, en invierno.

Para esta investigación, se utilizó residuo vegetal de maíz (Zea mays), el cual, fue cosechado, manualmente, en un cultivo contiguo al experimento, en mayo de 2007. Después de cosechado, el residuo, seco al aire, fue fraccionado en 
pedazos (triturador estacionario) y, en seguida, almacenado en un lugar seco y ventilado, hasta el momento de la instalación del experimento en campo, donde fue pesado y distribuido uniformemente en el área de cada tratamiento. Una vez distribuida la cobertura en el suelo, se aplicó, de forma manual, el nitrógeno y, a continuación, una irrigación equivalente a $6,0 \mathrm{~mm}$ de agua, con el objetivo de disolver el $\mathrm{N}$ y reducir sus pérdidas por la volatilización.

Durante el experimento, la superficie del suelo, en los tratamientos, fue mantenida libre de plantas dañinas, por medio de limpiezas manuales y se realizó control fitosanitario, empleando Cercobin 700 PM (Thiofanato Metílico), para el control de la Antracnosis (Colletotrichum indemuthianum), en la dosis de producto, recomendada comercialmente. Las parcelas fueron irrigadas con aspersión convencional, siendo que el manejo fue por tensiometría, de manera que se mantuvo en la capa de 0,0-0,2m, el contenido de agua en el suelo próximo a capacidad de campo correspondiente, durante todo el ciclo del cultivo. Se usó la humedad crítica de irrigación, el correspondiente a la tensión del suelo igual al potencial matricial $-35 \mathrm{kPa}$ en el tratamiento con cantidad de $4,0 \mathrm{t} \cdot \mathrm{ha}^{-1}$ de residuo.

La siembra del fríjol (Phaseolus vulgaris L.), variedad Carioca Precoce fue realizada el 10 de junio de 2007. La emergencia de las plántulas ocurrió ocho días después de la siembra, teniendo el desarrollo del cultivo establecido, durante el periodo de invierno. La cosecha fue realizada, manualmente, el 19 de septiembre de 2007, totalizando un ciclo de 95 días, a partir de la emergencia de las plántulas.

El monitoreo del contenido de agua en el suelo, se efectuó con la finalidad de controlar el sistema de irrigación y verificar el comportamiento de la humedad dentro de los tratamientos propuestos. El contenido de agua en el suelo fue determinado en tres puntos por parcela, en la profundidad de $0,20 \mathrm{~m}$, para un total de 108 muestras, tres veces por semana, aplicando el método gravimétrico de humedad actual (EMBRAPA, 1997).

Después de la cosecha, las muestras de suelo fueron colectadas en un punto por parcela, utilizando anillos volumétricos de $0,05 \mathrm{~m}$ de diámetro y $0,04 \mathrm{~m}$ de altura, en las profundidades de $0,00-0,05 m, 0,05-0,10 \mathrm{~m}$ y $0,10-0,15 \mathrm{~m}$, para un total de 108 muestras. La microporosidad, se determinó en mesa de tensión y correspondió a la humedad volumétrica de la muestra sometida a una tensión de $-0,006 \mathrm{MPa}$, una vez saturada (Oliveira, 1968). La porosidad total y la densidad del suelo fueron obtenidas por el método del anillo volumétrico, según EMBRAPA (1997) y, la macroporosidad, por diferencia entre la porosidad total y la microporosidad.
Para establecer la resistencia del suelo a la penetración fue importante evaluar la humedad del suelo, durante este análisis, por lo tanto, el contenido de agua en el suelo fue obtenido por el método gravimétrico (EMBRAPA, 1997), en las profundidades de $0,00-0,10 \mathrm{~m}, 0,10-0,20 \mathrm{~m}$ y $0,20-0,40 \mathrm{~m}$, en un punto por parcela (total de 108 muestras). Para la determinación de la resistencia del suelo a la penetración, se recurrió al penetrómetro de impacto modelo IAA/Planalsucar, con ángulo de cono de $30^{\circ}$, que fue insertado en el suelo hasta la profundidad de $0,45 \mathrm{~m}$, en un punto por parcela (un total de 36 puntos). La transformación de la penetración de la punta del aparato en el suelo ( $\mathrm{cm} /$ impacto) en resistencia a la penetración fue obtenida por la fórmula de Stolf (1991):

$$
R=\frac{M g+m g+\left(\frac{M}{M+m} * \frac{M g * h}{x}\right)}{A}
$$

donde $\mathrm{R}$, es la resistencia a la penetración, $\mathrm{kgf} \cdot \mathrm{cm}^{-2}\left(\mathrm{kgf} \cdot \mathrm{cm}^{-2}\right.$ * 0,098 = MPa); $M$, la masa del émbolo, $4 \mathrm{~kg}$ (ton $-4 \mathrm{kgf}$ ); $\mathrm{m}$, la masa del aparato sin émbolo, $3,2 \mathrm{~kg}$ ( $\mathrm{mg}-3,2 \mathrm{kgf}) ; \mathrm{h}$, la altura de caída del émbolo, $40 \mathrm{~cm}$; x, la penetración de la punta del aparato, cm/impacto, y A, el área de cono, $1,29 \mathrm{~cm}^{2}$.

Las lecturas de temperatura del suelo fueron obtenidas durante el desarrollo del cultivo de frijol en la profundidad de 0,15m y 0,30m, en dos períodos (9:00 horas y 14:00 horas), por medio del termómetro Soloterm 1200, efectuadas todos los días del ciclo del cultivo en seis puntos por parcela, para un total de 216 lecturas diarias por profundidad. La temperatura del aire fue obtenida mediante las lecturas detectadas por la estación meteorológica Campbell, localizada en el área experimental.

La productividad $\left(\mathrm{kg} \cdot \mathrm{ha}^{-1}\right)$ fue calculada utilizando los datos del área útil de cada parcela. Fueron evaluadas, en media, 30 plantas por $\mathrm{m}^{2}$ en el centro de cada parcela, de las cuales, fueron retiradas aleatoriamente tres plantas, para determinar el número de vainas por planta, el número de granos por vaina, materia seca total de la parte aérea en gramos, masa de 100 granos e índice de cosecha (IC), éste último, establecido por medio de la relación entre la masa de granos y la masa seca de toda la planta.

Los datos fueron sometidos a análisis de varianza para evaluación de los tratamientos y comparación de medias por el test de Tukey, con 95\% de confiabilidad de los datos, por medio del paquete estadístico SAS (Schlotzhaver E Littell, 1997). 


\section{RESULTADOS Y DISCUSIÓN}

Los datos de las medias de densidad del suelo, porosidad total, microporosidad y macroporosidad no presentaron diferencia significativa entre las diferentes profundidades y los tratamientos evaluados (Tabla 1), ya que el tiempo de implantación del sistema de siembra directa no fue suficiente, indicando, que tal vez, sea necesario un periodo de tiempo más prolongado de evaluación del experimento, para que la rotación de cultivos exprese su beneficio. Assis \& Lanças (2005) verificaron mejoría en las propiedades físicas del suelo, de acuerdo con el tiempo de adopción del sistema siembra directa, a partir de los doce años de implantación. Es necesario observar que los resultados relacionados con los sistemas de manejo del suelo presentan diversidad de respuestas a un mismo sistema, por causa de las características del suelo, de la planta y del clima, entre otros.

Algunos autores han observado valores superiores de microporosidad en el sistema de manejo de siembra directa, principalmente, próximo a la superficie, como Stone $\&$ Silveira (2001), Abreu et al. (2004) y Genro Júnior et al. (2004). Los valores de macroporosidad en la media de las diferentes profundidades y tratamientos evaluados fueron iguales $\mathrm{o}$ superiores de $0,10 \mathrm{~m}^{3} \cdot \mathrm{m}^{-3}$ (Tabla 1), mínimo adecuado para los intercambios líquidos y gaseosos y para el crecimiento de las raíces de la mayoría de los cultivos (Camargo \& Alleoni, 1997; Costa et al. 2003).

Para el análisis de resistencia del suelo a la penetración fue tomado como referencia solo las cantidades de cobertura, puesto que la cantidad de nitrógeno no presentó influencia en esta propiedad, por medio del análisis estadístico. Los tratamientos con aplicación de residuo de maíz mostraron menores valores de resistencia del suelo a la penetración en relación al estado inicial del área y al tratamiento sin aplicación de cobertura (Tabla 2). La disminución de los valores de resistencia del suelo a la penetración están relacionados a la manutención del contenido de agua en el suelo proporcionado por la cobertura, considerando que el contenido de agua en el suelo ejerce fuerte influencia en los resultados de esta característica (Stolf, 1991).

Se observa que a partir de la profundidad de 0,00-0,10m, los valores de resistencia del suelo a la penetración fueron menores en el tratamiento con aplicación de $10,0 \mathrm{t} \cdot \mathrm{ha}^{-1} \mathrm{de}$ residuo de maíz, siendo que este procedimiento presentó un mayor contenido de agua en el suelo en las diferentes profundidades evaluadas (Tabla 2). Los valores medios encontrados para la resistencia del suelo a la penetración

Tabla 1. Datos de las medias de densidad del suelo $\left(\mathrm{kg} \cdot \mathrm{dm}^{-3}\right)$, porosidad total $\left(\mathrm{m}^{3} \cdot \mathrm{m}^{-3}\right)$, microporosidad $\left(\mathrm{m}^{3} \cdot \mathrm{m}^{-3}\right)$ y macroporosidad del suelo $\left(\mathrm{m}^{3} \cdot \mathrm{m}^{-3}\right)$ en las diferentes profundidades estudiadas $(n=3)$.

\begin{tabular}{|c|c|c|c|c|c|c|c|c|c|c|c|c|}
\hline \multirow{4}{*}{$\begin{array}{c}\text { Tratamiento } \\
\left(\mathrm{kg} \cdot \mathrm{ha}^{-1}-\right. \\
\left.\mathrm{t} \cdot \mathrm{ha}^{-1}\right)\end{array}$} & \multicolumn{12}{|c|}{ Profundidad (m) } \\
\hline & \multicolumn{4}{|c|}{$0,00-0,05$} & \multicolumn{4}{|c|}{$0,05-0,10$} & \multicolumn{4}{|c|}{$0,10-0,15$} \\
\hline & \multicolumn{3}{|c|}{ Porosidad } & \multirow{2}{*}{$\frac{\text { DS }}{-}$} & \multicolumn{3}{|c|}{ Porosidad } & \multirow{2}{*}{$\frac{D S}{-}$} & \multicolumn{3}{|c|}{ Porosidad } & DS \\
\hline & Total & micro & macro & & Total & Micro & macro & & Total & micro & macro & - \\
\hline $0,0-0,0$ & 0,52 & 0,42 & 0,10 & 1,34 & 0,50 & 0,37 & 0,13 & 1,28 & 0,51 & 0,40 & 0,11 & 1,30 \\
\hline $0,0-4,0$ & 0,51 & 0,42 & 0,09 & 1,33 & 0,47 & 0,37 & 0,11 & 1,30 & 0,50 & 0,40 & 0,10 & 1,30 \\
\hline $0,0-6,0$ & 0,50 & 0,42 & 0,08 & 1,32 & 0,50 & 0,41 & 0,09 & 1,30 & 0,51 & 0,40 & 0,11 & 1,32 \\
\hline $0,0-10,0$ & 0,50 & 0,40 & 0,10 & 1,29 & 0,50 & 0,39 & 0,11 & 1,26 & 0,49 & 0,40 & 0,09 & 1,29 \\
\hline $50-0,0$ & 0,42 & 0,35 & 0,07 & 1,31 & 0,51 & 0,41 & 0,10 & 1,30 & 0,50 & 0,41 & 0,09 & 1,36 \\
\hline $50-4,0$ & 0,50 & 0,40 & 0,10 & 1,23 & 0,52 & 0,42 & 0,10 & 1,29 & 0,52 & 0,42 & 0,10 & 1,28 \\
\hline $50-6,0$ & 0,51 & 0,42 & 0,09 & 1,41 & 0,51 & 0,41 & 0,10 & 1,28 & 0,52 & 0,42 & 0,10 & 1,30 \\
\hline $50-10,0$ & 0,51 & 0,40 & 0,11 & 1,24 & 0,52 & 0,40 & 0,12 & 1,32 & 0,51 & 0,40 & 0,11 & 1,29 \\
\hline $120-0,0$ & 0,53 & 0,41 & 0,11 & 1,37 & 0,50 & 0,41 & 0,08 & 1,29 & 0,48 & 0,38 & 0,10 & 1,31 \\
\hline $120-4,0$ & 0,52 & 0,39 & 0,13 & 1,26 & 0,53 & 0,39 & 0,14 & 1,31 & 0,50 & 0,41 & 0,09 & 1,33 \\
\hline $120-6,0$ & 0,51 & 0,41 & 0,11 & 1,30 & 0,53 & 0,40 & 0,13 & 1,26 & 0,53 & 0,40 & 0,13 & 1,28 \\
\hline $120-10,0$ & 0,50 & 0,38 & 0,12 & 1,26 & 0,50 & 0,41 & 0,09 & 1,37 & 0,49 & 0,41 & 0,09 & 1,31 \\
\hline Media & 0,50 & 0,40 & 0,10 & 1,31 & 0,51 & 0,40 & 0,11 & 1,29 & 0,50 & 0,40 & 0,10 & 1,30 \\
\hline CV \% & 8,9 & 9,9 & 27,7 & 8,8 & 5,2 & 7,5 & 23,9 & 3,7 & 5,3 & 4,9 & 22,4 & 3,4 \\
\hline
\end{tabular}


Tabla 2. Valores medios de la resistencia del suelo a la penetración y contenido de agua en el suelo en las diferentes profundidades estudiadas $(n=9)$.

\begin{tabular}{|c|c|c|c|c|c|c|c|c|}
\hline \multirow{2}{*}{ Profundidad $(\mathrm{m})$} & \multicolumn{3}{|c|}{ Resistencia del suelo a la Penetración (MPa) } & \multicolumn{4}{|c|}{ Contenido de agua en el suelo $\left(\mathbf{g}^{\cdot} \mathbf{g}^{-1}\right.$ ) } \\
\cline { 2 - 9 } & $0,0 \mathrm{t} \cdot \mathrm{ha}^{-1}$ & $4,0 \mathrm{t} \cdot \mathrm{ha}^{-1}$ & $6,0 \mathrm{t} \cdot \mathrm{ha}^{-1}$ & $10,0 \mathrm{t} \cdot \mathrm{ha}^{-1}$ & $0,0 \mathrm{t} \cdot \mathrm{ha}^{-1}$ & $4,0 \mathrm{t} \cdot \mathrm{ha}^{-1}$ & $6,0 \mathrm{t} \cdot \mathrm{ha}^{-1}$ & $10,0 \mathrm{t} \cdot \mathrm{ha}^{-1}$ \\
\hline $0,00-0,05$ & $0,88 \mathrm{a}$ & $0,82 \mathrm{a}$ & $0,67 \mathrm{a}$ & $0,70 \mathrm{a}$ & $0,25 \mathrm{c}$ & $0,26 \mathrm{bc}$ & $0,27 \mathrm{ab}$ & $0,28 \mathrm{a}$ \\
\hline $0,05-0,10$ & $2,69 \mathrm{a}$ & $2,10 \mathrm{a}$ & $1,89 \mathrm{a}$ & $1,82 \mathrm{a}$ & $0,25 \mathrm{c}$ & $0,26 \mathrm{bc}$ & $0,27 \mathrm{ab}$ & $0,28 \mathrm{a}$ \\
\hline $0,10-0,15$ & $3,98 \mathrm{a}$ & $2,93 \mathrm{a}$ & $3,13 \mathrm{a}$ & $2,63 \mathrm{a}$ & $0,23 \mathrm{c}$ & $0,24 \mathrm{bc}$ & $0,25 \mathrm{~b}$ & $0,27 \mathrm{a}$ \\
\hline $0,15-0,20$ & $6,46 \mathrm{a}$ & $3,91 \mathrm{~b}$ & $4,51 \mathrm{ab}$ & $2,91 \mathrm{~b}$ & $0,22 \mathrm{c}$ & $0,24 \mathrm{bc}$ & $0,25 \mathrm{ab}$ & $0,26 \mathrm{a}$ \\
\hline $0,20-0,25$ & $7,12 \mathrm{a}$ & $4,60 \mathrm{~b}$ & $4,26 \mathrm{~b}$ & $3,88 \mathrm{~b}$ & $0,22 \mathrm{c}$ & $0,24 \mathrm{~b}$ & $0,25 \mathrm{ab}$ & $0,26 \mathrm{a}$ \\
\hline $0,25-0,30$ & $5,68 \mathrm{a}$ & $4,31 \mathrm{ab}$ & $3,84 \mathrm{ab}$ & $3,36 \mathrm{~b}$ & $0,22 \mathrm{~b}$ & $0,24 \mathrm{a}$ & $0,25 \mathrm{a}$ & $0,25 \mathrm{a}$ \\
\hline $0,30-0,35$ & $4,31 \mathrm{a}$ & $3,90 \mathrm{a}$ & $3,60 \mathrm{a}$ & $3,00 \mathrm{a}$ & $0,22 \mathrm{~b}$ & $0,24 \mathrm{a}$ & $0,25 \mathrm{a}$ & $0,25 \mathrm{a}$ \\
\hline $0,35-0,40$ & $3,84 \mathrm{a}$ & $3,18 \mathrm{a}$ & $2,53 \mathrm{a}$ & $2,32 \mathrm{a}$ & $0,22 \mathrm{~b}$ & $0,24 \mathrm{a}$ & $0,25 \mathrm{a}$ & $0,25 \mathrm{a}$ \\
\hline $0,40-0,45$ & $1,29 \mathrm{a}$ & $1,00 \mathrm{a}$ & $0,96 \mathrm{a}$ & $0,89 \mathrm{a}$ & $0,22 \mathrm{~b}$ & $0,24 \mathrm{a}$ & $0,25 \mathrm{a}$ & $0,25 \mathrm{a}$ \\
\hline $\mathrm{CV}(\%)$ & 66,2 & 59,6 & 64,9 & 58,8 & 9,4 & 6,8 & 8,7 & 8,0 \\
\hline
\end{tabular}

Medias seguidas por la misma letra en las filas no difieren entre sí por el test de Tukey a 5\% de probabilidad.

están comprendidos en la clase alta en las profundidades en estudio, de acuerdo con Arshad et al. (1996).

Los datos de la resistencia del suelo a la penetración presentaron diferencia entre las medias en los diferentes tratamientos, reflejando influencia de la cantidad de cobertura a lo largo del perfil (Tabla 2). Con relación al contenido de agua en el suelo, a partir de la profundidad de $0,25 \mathrm{~m}$, sólo se registra diferencia significativa con relación al tratamiento sin presencia de cobertura, pues la manutención del contenido de agua en el suelo fue influenciada por la cantidad de cobertura en las profundidades iniciales. Para el cultivo de fríjol esto es importante, pues gran cantidad del sistema radicular de este cultivo explora esta profundidad. Resultados obtenidos por Pires et al. (1991) para fines de manejo de irrigación, en un tipo de suelo, observaron que es adecuado considerar la profundidad efectiva del sistema radicular de $0,30 \mathrm{~m}$ para el fríjol, donde se concentra más del $80 \%$ de las raíces finas. A partir de esa profundidad ocurre una drástica reducción en la distribución porcentual de las raíces.

Los valores elevados de resistencia del suelo a la penetración en la profundidad de $0,15-0,30 \mathrm{~m}$ (Tabla 2), en los diferentes tratamientos estudiados, puede no comprometer al cultivo instalado, debido a la mayor continuidad de poros, de homogeneidad del suelo y a la mayor actividad microbiana que, normalmente, ocurren en la siembra directa (Arshad et al. 1996). Ehlers et al. (1983) relatan que valores de resistencia del suelo a la penetración en el orden de 5,0MPa son admitidos en siembra directa, pues las raíces crecen por canales continuos dejados por la fauna del suelo y por el sistema radicular descompuesto. Wutke et al. (2000) registraron medias máximas de resistencia del suelo a la penetración de 5,0 a 7,0MPa, entre las profundidades de 0,20 a $0,35 \mathrm{~m}$, para el fríjol irrigado sobre diferentes rotaciones de cultivos, en tres años de ensayo.

La compactación del suelo es más perjudicial en suelos secos, mientras que, en condiciones de mayor contenido de agua, puede haber crecimiento radicular en valores de resistencia del suelo a la penetración, superiores a 4,0MPa (Dexter, 1987). Por tanto, la manutención de la cobertura es fundamental para un mayor desenvolvimiento del sistema radicular y aumento de la productividad del cultivo de fríjol.

Los valores medios del contenido de agua en el suelo son expuestos en la tabla 3. Se observa que no hay diferencia significativa al comparar cada cantidad de cobertura en las distintas dosis de nitrógeno, pero sí registra diferencias significativas al comparar la misma dosis de nitrógeno en las cantidades de cobertura. Al analizar las diferencias significativas de los valores medios, se verifica que a mayor la cantidad de cobertura, mayor es el contenido de agua en el suelo. Derpsch et al. (1991) verificaron que la mayor disponibilidad de agua sobre siembra directa se debe, principalmente, a la diminución de las pérdidas por evaporación y al aumento de la tasa de infiltración en función de la cobertura del suelo. Melo Filho E Silva (1993), en maíz y Stone \& Silveira (1999), en frijol, verificaron mayor economía de agua en el sistema siembra directa, en comparación a otros sistemas de labranza, sin cobertura. 
Tabla 3. Valores medios del contenido de agua $(n=240)$ y de la amplitud térmica del suelo en las diferentes profundidades $(n=33)$ teniendo en cuenta los tratamientos estudiados a lo largo del ciclo del cultivo de frijol irrigado.

\begin{tabular}{|c|c|c|c|c|}
\hline \multirow{2}{*}{ Tratamiento } & \multicolumn{4}{|c|}{ Humedad Gravimétrica $\left(\mathbf{g} \cdot \mathbf{g}^{-1}\right)$} \\
\hline & 0,0 ton ha $^{-1}$ & 4,0 ton ha $^{-1}$ & 6,0 ton ha $^{-1}$ & 10,0 ton $\cdot$ ha $^{-1}$ \\
\hline $0,0 \mathrm{~kg} \cdot \mathrm{ha}^{-1} \mathrm{~N}$ & $0,23 \mathrm{bA}$ & $0,25 a b A$ & $0,25 \mathrm{aA}$ & $0,27 \mathrm{aA}$ \\
\hline $50,0 \mathrm{~kg} \cdot h \mathrm{a}^{-1} \mathrm{~N}$ & $0,23 \mathrm{bA}$ & $0,24 a b A$ & $0,26 \mathrm{aA}$ & $0,27 \mathrm{aA}$ \\
\hline $120,0 \mathrm{~kg} \cdot \mathrm{ha}^{-1} \mathrm{~N}$ & $0,24 \mathrm{aA}$ & $0,25 \mathrm{aA}$ & $0,25 \mathrm{aA}$ & $0,25 \mathrm{aA}$ \\
\hline Media & $0,24 \mathrm{c}$ & $0,25 b$ & $0,25 a b$ & $0,26 \mathrm{a}$ \\
\hline \multirow[t]{2}{*}{ CV $(\%)$} & 15,0 & 12,7 & 12,2 & 11,1 \\
\hline & \multicolumn{4}{|c|}{ Amplitud térmica $\left({ }^{\circ} \mathrm{C}\right)$ del suelo en la profundidad de $0,15 \mathrm{~m}$} \\
\hline $0,0 \mathrm{~kg} \cdot \mathrm{ha}^{-1} \mathrm{~N}$ & $2,47 \mathrm{aA}$ & $1,55 \mathrm{bA}$ & $1,51 \mathrm{bA}$ & $1,26 \mathrm{bA}$ \\
\hline $50,0 \mathrm{~kg} \cdot h \mathrm{a}^{-1} \mathrm{~N}$ & $2,07 \mathrm{aAB}$ & $1,68 a b A$ & $1,45 \mathrm{bA}$ & $1,18 \mathrm{bA}$ \\
\hline $120,0 \mathrm{~kg} \cdot \mathrm{ha}^{-1} \mathrm{~N}$ & $1,92 \mathrm{aB}$ & $1,44 a b A$ & $1,62 \mathrm{abA}$ & $1,33 \mathrm{bA}$ \\
\hline \multirow[t]{2}{*}{ CV \% } & 38,9 & 38,8 & 40,8 & 34,3 \\
\hline & \multicolumn{4}{|c|}{ Amplitud térmica $\left({ }^{\circ} \mathrm{C}\right)$ del suelo en la profundidad de $0,30 \mathrm{~m}$} \\
\hline $0,0 \mathrm{~kg} \cdot \mathrm{ha}^{-1} \mathrm{~N}$ & $0,91 \mathrm{aA}$ & $0,88 \mathrm{aA}$ & $0,91 \mathrm{aA}$ & $0,85 \mathrm{aA}$ \\
\hline $50,0 \mathrm{~kg} \cdot h \mathrm{~h}^{-1} \mathrm{~N}$ & $0,96 \mathrm{aA}$ & $0,88 \mathrm{aA}$ & $0,81 \mathrm{aA}$ & $0,87 \mathrm{aA}$ \\
\hline $120,0 \mathrm{~kg} \cdot \mathrm{ha}^{-1} \mathrm{~N}$ & $0,91 \mathrm{aA}$ & $0,88 \mathrm{aA}$ & $0,94 \mathrm{aA}$ & $0,91 \mathrm{aA}$ \\
\hline CV $(\%)$ & 49,2 & 44,8 & 42,5 & 43,1 \\
\hline
\end{tabular}

Medias seguidas por la misma letra minúscula en las filas y mayúscula en las columnas, no difieren entre sí por el test de Tukey a 5\% de probabilidad.

Durante el ciclo del cultivo de frijol es posible verificar que la manutención del contenido de agua en el suelo es mayor en el tratamiento de $10,0 \mathrm{t} \cdot \mathrm{ha}^{-1}$ de cobertura (Figura 1). Según Stone \& Moreira (2000) para el cultivo de fríjol, el contenido de agua en el suelo mínimo para el adecuado crecimiento y buena productividad es de $0,23 \mathrm{~g} \cdot \mathrm{g}^{-1}$ y se observa que los tratamientos con cobertura, 4,0; 6,0 y 10,0t $\cdot$ ha $^{-1}$, quedaron por encima de ese requerimiento, a lo largo del ciclo del cultivo de fríjol.

Los valores medios de las amplitudes térmicas presentan diferencias significativas sólo en la profundidad de $0,15 \mathrm{~m}$, al evaluar las cantidades de cobertura del suelo. Estos valores no tienen interferencia de las dosis de nitrógeno (Tabla 3). Según Varejão-Silva (2001), la variación diaria de la temperatura del suelo depende del tipo de cobertura presente en la superficie, ya que ésta interfiere en el suplemento de energía proveniente del sol. Para una clase dada de suelo, la amplitud térmica diaria, a una determinada profundidad, con algún tipo de cobertura, contribuye para reducir sensiblemente la amplitud térmica diaria del suelo.
$\mathrm{Al}$ analizar los datos de amplitud térmica del suelo en las diferentes profundidades, se destaca que hay diferencias significativas al comparar las diferentes cantidades de cobertura para una misma dosis, en la profundidad de $0,15 \mathrm{~m}$. Se constató que el tratamiento con mayor cantidad de residuo en la superficie del suelo presentó menores amplitudes térmicas de temperatura a lo largo del ciclo del cultivo. Para la profundidad de $0,30 \mathrm{~m}$, se detectó que no hay un efecto directo de la cobertura, al no haber diferencias significativas en los datos (Tabla 3). El efecto de la cobertura en la superficie evitó que el suelo se calentara en proporciones iguales al suelo de área sin cobertura. Tales datos destacan la importancia de la cobertura vegetal en la disminución de la temperatura máxima del suelo, notándose que la cobertura registra baja conductividad térmica y alta reflectividad de los rayos solares (Oliveira et al. 2005). Trevisan et al. (2002) verificaron que la cobertura de avena disminuyó la amplitud térmica a 0,10 y a $0,20 \mathrm{~m}$ de profundidad durante todo el año, comparada con el suelo que permaneció sin cobertura. 


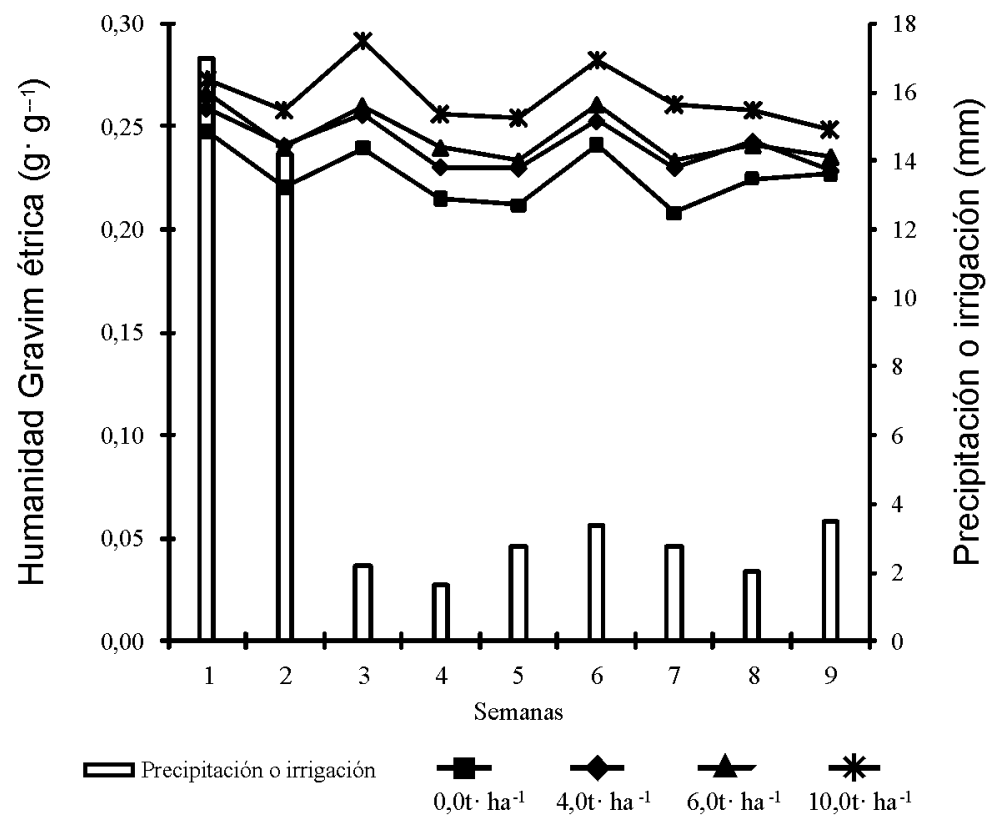

Figura 1. Contenido de agua en el suelo en los diferentes tratamientos a lo largo del ciclo del cultivo de frijol irrigado en la profundidad de 0,20 m.

Los resultados de temperatura del suelo están coherentes con los datos del contenido de agua, pues en sistemas de manejo conservacionistas, que mantienen los residuos en la superficie del suelo, reducen las temperaturas máximas y la amplitud diaria, con reflejos positivos en la conservación del contenido de agua en el suelo. Resultados semejantes fueron observados por Bragagnolo \& Mielniczuk (1990). Normalmente, en los suelos cultivados en sistemas conservacionistas, con residuos mantenidos en la superficie, la temperatura máxima y la amplitud térmica son menores, manifestándose en la humedad, en especial, en los primeros centímetros del suelo, de acuerdo a lo mencionado por Salton \& Mielniczuk (1995) y Costa et al. (2003).

La producción total presenta diferencia significativa en los tratamientos siendo que los mayores valores, correspondieron al tratamiento con mayor cantidad de nitrógeno aplicado $\left(120 \mathrm{~kg} \cdot \mathrm{ha}^{-1}\right)$ y mayor cantidad de cobertura $\left(10,0 \mathrm{t} \cdot \mathrm{ha}^{-1}\right)$ (Tabla 4). No se presentó diferencias significativas para las demás dosis de residuo y cantidades de nitrógeno. Con relación al número de plantas por $\mathrm{m}^{2}$ y la masa de 100 granos, sólo muestran diferencia significativa en relación a la cantidad de cobertura. El número de vainas por planta no registró diferencia entre los tratamientos. Resultados semejantes fueron encontrados por Andrade et al. (2002), quienes observaron, para el número de vainas, valores de 11,7 para fríjol cultivado en siembra directa con $100 \%$ de cobertura del suelo y superiores a los obtenidos por Medeiros et al. (2000), con la variedad IAC Carioca, que obtuvieron 10,1 vainas por planta.

Los valores de la masa de la parte aérea, masa de la parte aérea total y el índice de cosecha, solo presentaron diferencia significativa, considerando la cantidad de nitrógeno (Tabla 4). Los valores de número de granos por vaina y masa de granos total no presentaron diferencia significativa, en los tratamientos estudiados. Los resultados de número de granos por vaina encontrados por Medeiros et al. (2000) tampoco arrojaron diferencias significativas para los tratamientos estudiados, teniendo valores entre 4,1 a 4,5 , siendo estos inferiores a los hallados en este experimento.

Estos resultados expuestos permitieron identificar el comportamiento de las diferentes propiedades del suelo, así como las relaciones existentes entre ellas. Mediante la implementación de la siembra directa y uso de coberturas, se contribuye a futuras investigaciones, ya que este sistema permite la recuperación de suelos degradados físicamente, aunque es importante resaltar que los efectos de esta implementación son a largo plazo, siendo de gran interés la evaluación de dichas propiedades durante varios ciclos de cultivos. 
Tabla 4. Valores medios de los componentes de la producción del cultivo de frijol irrigado en los diferentes tratamientos estudiados $(n=12)$.

\begin{tabular}{|c|c|c|c|c|c|c|c|c|c|}
\hline \multirow{3}{*}{ Tratamiento } & \multicolumn{9}{|c|}{ Componentes de la producción del frijol irrigado } \\
\hline & $\mathrm{Pt}$ & NP & $\mathrm{Pg}$ & Nv & Ppa & $\mathrm{Ngv}$ & Ppat & Pgt & IC \\
\hline & $\left(\mathrm{kg} \cdot \mathrm{ha}^{-1}\right)$ & - & (g) & - & (g) & - & (g) & (g) & - \\
\hline \multicolumn{10}{|c|}{ Cantidades de nitrógeno aplicado } \\
\hline $0,0 \mathrm{~kg} \cdot \mathrm{ha}^{-1}$ & $2327 \mathrm{~b}$ & $29,6 \mathrm{a}$ & $22,7 \mathrm{a}$ & $12,0 \mathrm{a}$ & $7,9 a b$ & $5,1 \mathrm{a}$ & $27,9 \mathrm{ab}$ & $42,0 \mathrm{a}$ & $63,6 \mathrm{a}$ \\
\hline $50,0 \mathrm{~kg} \cdot \mathrm{ha}^{-1}$ & $2613 b$ & $31,8 \mathrm{a}$ & $21,9 \mathrm{a}$ & $10,5 \mathrm{a}$ & $6,9 \mathrm{~b}$ & $5,0 \mathrm{a}$ & $20,8 b$ & $34,5 \mathrm{a}$ & $62,1 \mathrm{ab}$ \\
\hline $120,0 \mathrm{~kg} \cdot \mathrm{ha}^{-1}$ & $3287 \mathrm{a}$ & $30,6 \mathrm{a}$ & $21,6 \mathrm{a}$ & $13,3 \mathrm{a}$ & $9,6 \mathrm{a}$ & $4,8 \mathrm{a}$ & $28,7 \mathrm{a}$ & $43,3 a$ & $59,7 b$ \\
\hline \multirow{2}{*}{ CV (\%) } & 21,6 & 17,1 & 3,9 & 25,3 & 25,2 & 8,0 & 25,2 & 31,2 & 4,0 \\
\hline & \multicolumn{9}{|c|}{ Cantidades de residuos de maíz } \\
\hline $0,0 \mathrm{t} \cdot \mathrm{ha}^{-1}$ & $2400 \mathrm{~b}$ & $30,9 a b$ & $21,2 b$ & $10,8 \mathrm{a}$ & $6,9 a$ & $4,9 a$ & $20,9 a$ & $356 a$ & $62,9 a$ \\
\hline $4,0 \mathrm{t} \cdot \mathrm{ha}^{-1}$ & $2713 a b$ & $27,7 \mathrm{~b}$ & $22,2 a b$ & $12,8 \mathrm{a}$ & $8,8 \mathrm{a}$ & $4,7 \mathrm{a}$ & $26,5 \mathrm{a}$ & $41,1 \mathrm{a}$ & $60,8 \mathrm{a}$ \\
\hline $6,0 \mathrm{t} \cdot \mathrm{ha}^{-1}$ & $2700 a b$ & $30,0 a b$ & $22,4 \mathrm{a}$ & $11,9 a$ & $8,3 a$ & $5,2 a$ & $24,8 \mathrm{a}$ & $40,5 a$ & $61,1 \mathrm{a}$ \\
\hline $10,0 \mathrm{t} \cdot \mathrm{ha}^{-1}$ & 3167 a & $34,1 \mathrm{a}$ & $22,0 \mathrm{ab}$ & $12,2 \mathrm{a}$ & $8,5 a$ & $5,0 \mathrm{a}$ & $25,6 \mathrm{a}$ & $42,7 \mathrm{a}$ & $62,2 \mathrm{a}$ \\
\hline CV (\%) & 24,6 & 16,5 & 3,6 & 25,9 & 25,8 & 7,5 & 25,8 & 30,2 & 4,6 \\
\hline
\end{tabular}

Medias seguidas por la misma letra en las columnas no difieren entre sí por el test de Tukey a $5 \%$ de probabilidad. $\mathrm{Pt}=$ Producción total; $\mathrm{NP}=$ Número de plantas por $\mathrm{m}^{2} ; \mathrm{Pg}=$ Masa de 100 granos; $\mathrm{Nv}=$ Número de vainas por planta; $\mathrm{Ppa}=$ Masa de la parte aérea; $\mathrm{Ngv}=$ Número de granos por vaina; $\mathrm{Ppat}=$ Masa de la parte aérea total;

Pgt = Masa granos total; IC = Índice de cosecha.

Conflictos de interés: La investigación y el manuscrito, se realizaron con la colaboración de todos los autores, quienes declaramos que no existe ningún conflicto de intereses que ponga en riesgo la validez de los resultados presentados.

\section{BIBLIOGRAFÍA}

1. ABREU, S.L.; REICHERT, J.M.; REINERT, D.J. 2004. Escarificação mecânica e biológica para a redução da compactação em argissolo franco-arenoso sob plantio direto. Rev. Bras. Ciência do Solo (Viçosa). 28(3):519-531.

2. ANDRADE, R.S.; MOREIRA, J.A.A.; STONE, L.F.; CARVALHO, J.A. 2002. Consumo relativo de água do feijoeiro no plantio direto em função da porcentagem de cobertura morta do solo. Rev. Bras. Engenharia Agrícola e Ambiental (Campina Grande). 6(1):35-38.

3. ARSHAD, M.A.; LOWER, B.; GROSSMAN, B. 1996. Physical tests for monitoring soil quality. In: DORAN, J.W.; JONES, A.J. eds. Methods for assessing soil quality. Ed. Soil Science Society of America (Madison). (Special Publication, 49) p.123-141.
4. ASSIS, R.L.; LANÇAS, K.P. 2005. Avaliação dos atributos físicos de um Nitossolo Vermelho distroférrico sob sistema plantio direto, preparo convencional e mata nativa. Rev. Bras. Ciência do Solo (Viçosa). 29(4):515-522.

5. BRAGAGNOLO, N.; MIELNIKZUK, L. 1990. Cobertura do solo por palha de trigo e seu relacionamento com a temperatura e umidade do solo. Rev. Bras. Ciência do Solo (Campinas). 14(3):369-374.

6. CAMARGO, O.A.; ALLEONI, L.R.F. 1997. Compactação do solo e o desenvolvimento das plantas. Ed. ESALQ (Piracicaba). 132p.

7. CAMPOS, B.C.; REINERT, D.J.; NICOLODI, R.; RUEDELL, J.; PETRERE, C. 1995. Estabilidade estrutural de um Latossolo Vermelho-Escuro distrófico após sete anos de rotação de culturas e sistemas de manejo de solo. Rev. Bras. Ciência do Solo (Campinas). 19(1):121-126.

8. COSTA, F.S.; ALBUQUERQUE, J.A.; BAYER, C.; FONTOURA, S.M.V.; WOBETO, C. 2003. Propriedades físicas de um Latossolo Bruno afetadas pelos sistemas plantio direto e preparo convencional. Rev. Bras. Ciência do Solo (Viçosa). 27(3):527-535. 
9. DERPSCH, R.; ROTH, C.H.; SIDIRAS, N.; KOPKE, U. 1991. Controle da erosão no Paraná, Brasil. Em: Sistemas de cobertura do solo, plantio direto e preparo conservacionista do solo. Ed. GTZ (Eschborn). 272p.

10. DEXTER, A.R. 1987. Mechanics of root growth. Plant and Soil (Dordrecht). 98(3):303-312.

11. EHLERS, W.; KOPKE, V.; HESSE, F.; BÖHM, W. 1983. Penetration resistance and root growth of oats in tilled and untilled loess soil. Soil and Tillage Research (Amsterdam). 3(3):261-275.

12. EMBRAPA, Centro Nacional de Pesquisa de Solos. 1997. Manual de métodos de análise de solo. Ed. EMBRAPA, CNPS (Rio de Janeiro). 212p.

13. EMBRAPA, Centro Nacional de Pesquisa de Solos. 1999. Sistema Brasileiro de Classificação de Solos. Ed. Embrapa. Produção de Informação (Rio de Janeiro) e Embrapa Solos (Brasília). 412p.

14. FURLANI, C.E.A.; GAMERO, C.A.; LEVIEN, R.; SILVA, R.P.; CORTEZ, J.W. 2008. Temperatura do solo em função do preparo do solo e do manejo da cobertura de inverno. Rev. Bras. Ciência do Solo (Viçosa). 32(1):375-380.

15. GENRO JÙNIOR, S.A.; REINERT, D.J.; REICHERT, J.M. 2004. Variabilidade temporal da resistência à penetração de um LATOSSOLO Argiloso sob semeadura direta com rotação de culturas. Rev. Bras. Ciência de Solo (Viçosa). 28(3):477-484.

16. KÖPPEN, W. 1936. Das geographische System der Klimate. In: Handbuch der Klimatologie. Köppen, W.; Geiger, G., eds. Gebr. Borntraeger. p.1-44.

17. LAL, R. 1974. Effect of constant and fluctuating soil temperature on the growth, development and nutrient uptake of maize seedlings. Plant and Soil (Dordrecht). 40(3):589-606.

18. MEDEIROS, G.A.; ARRUDA, F.B.; SAKAI, E.; FUJIWARA, M.; BONI, N.R. 2000. Crescimento vegetativo e coeficiente de cultura do feijoeiro relacionados a graus-dia acumulados. Pesquisa Agropecuária Brasileira (Brasília). 35(9):1733-1742.

19. MELO FILHO, J.F.; SILVA, J.R.C. 1993. Erosão, teor de água no solo e produtividade do milho em plantio direto e preparo convencional de um Podzólico Vermelho-Amarelo no Ceará. Rev. Bras. Ciência do Solo (Campinas). 17(2):291-297.
20. OLIVEIRA, L.B. 1968. Determinação da macro e microporosidade pela "mesa de tensão" em amostras de solo com estrutura indeformada. Pesquisa Agropecuária Brasileira (Brasília). 3:197-200.

21. OLIVEIRA, M.L.; RUIZ, H.A.; COSTA, L.M.; SCHAEFER, C.E.G.R. 2005. Flutuações de temperatura e umidade do solo em resposta à cobertura vegetal. Rev. Bras. Engenharia Agrícola e Ambiental (Campina Grande). 9(4):535-539.

22. PIRES, R.C.M.; ARRUDA, F.B.; FUJIWARA, M.; SAKAI, E.; BORTOLETTO N. 1991. Profundidade do sistema radicular das culturas de feijão e trigo sob pivô central. Bragantia (Campinas). 50(1):153-162.

23. SALTON, J.C.; MIELNICZUK, J. 1995. Relações entre sistemas de preparo, temperatura e umidade de um Podzólico Vermelho Escuro de Eldorado do Sul (RS). Rev. Bras. Ciência do Solo (Campinas). 19(2):313-319.

24. SATURNINO, H.M.; LANDERS, J.N. 1997. O meio ambiente e o plantio direto. Ed. APDC (Goiânia). 18p.

25. SCHLOTZHAVER, S.D.; LITTELL, R.C. 1997. SAS: system for elementary statistical analysis. Ed. Cary. 905p.

26. STOLF, R. 1991. Teoria e teste experimental de fórmulas de transformação dos dados de penetrômetro de impacto em resistência do solo. Rev. Bras. Ciência do Solo (Campinas). 15(3):229-235.

27. STONE, L.F.; SILVEIRA, P.M. 1999. Efeitos do sistema de preparo na compactação do solo, disponibilidade hídrica e comportamento do feijoeiro. Pesquisa Agropecuária Brasileira (Brasília). 34(1):83-91.

28. STONE, L.F.; MOREIRA, J.A.A. 2000. Efeitos de sistemas de preparo do solo no uso da água e na produtividade do feijoeiro. Pesquisa Agropecuária Brasileira (Brasília). 35(4):835-841.

29. STONE, L.F., SILVEIRA, P.M. 2001. Efeitos do sistema de preparo e da rotação de culturas na porosidade e densidade do solo. Rev. Bras. Ciência do Solo (Viçosa). 25(2):395-401.

30. TREVISAN, R.; HERTER, F.G.; PEREIRA, I.S. 2002. Variação da amplitude térmica do solo em pomar de pessegueiro cultivado com aveia preta (Avena sp.) e em sistema convencional. Rev. Bras. Agrociência (Pelotas). 8(2):155-157. 
31. VAREJÃO-SILVA, M.A. 2001. Meteorologia e Climatologia.

Ed. Pax (Brasília). p.101-108.

32. WUTKE, E.B., ARRUDA, F.B., FANCELLI, A.L., PEREIRA, J.C.V.N.A., SAKAI, E., FUJIWARA, M. AMBROSANO, G.M.B. 2000. Propriedades do solo e sistema radicular do feijoeiro irrigado em rotação de culturas. Rev. Bras. Ciência do Solo (Viçosa). 24(3):621-633.

Recibido: Febrero 22 de 2010

Aceptado: Octubre 13 de 2010 